\title{
Characterization of Surface Electromyography Patterns of Healthy and Incomplete Spinal Cord Injury Subjects Interacting with an Upper-Extremity Exoskeleton
}

\author{
Craig G. McDonald, Troy A. Dennis, and Marcia K. O’Malley
}

\begin{abstract}
Rehabilitation exoskeletons may make use of myoelectric control to restore in patients with significant motor impairment following a spinal cord injury (SCI) a sense of volitional control over their limb - a crucial component for recovery of movement. Little investigation has been done into the feasibility of using surface electromyography (sEMG) as an exoskeleton control interface for SCI patients, whose impairment manifests in a highly variable way across the patient population. We have demonstrated that by using only a small subset of features extracted from eight bipolar electrodes recording on the upper arm and forearm muscles, we can achieve high predictive accuracy for the intended direction of motion. Five healthy subjects and two SCI subjects performed voluntary isometric contractions while wearing an exoskeleton for the wrist and elbow joints, generating six distinct single and multi-DoF motions in a total of sixteen possible directions. Using linear discriminant analysis, classification performance was then evaluated using randomly selected holdout test data from the same recording session. Commonalities across subjects, both healthy and SCI, were analyzed at the levels of selected features and the values of commonly selected features. Future work will be to investigate group-specific classification of SCI subjects' intended movements for use in the real-time control of a rehabilitation exoskeleton.
\end{abstract}

\section{INTRODUCTION}

Rehabilitation after spinal cord injury (SCI) is largely composed of repetitive movements over long time periods with the goal of inducing plasticity in the nervous system. Such training has been shown to increase muscle strength and coordination [1], [2]. Rice University's Mechatronics and Haptic Interfaces (MAHI) Lab has recently demonstrated the feasibility of their rehabilitation exoskeletons for facilitating motor recovery after incomplete SCI [3]. Despite these successes, the reach of this work is limited to individuals with mild to moderate impairment, since participants must initiate and sustain movement in order to realize the greatest benefit from the therapy.

One popular technique for measuring the voluntary muscle contractions of individuals, healthy or otherwise, is surface electromyography (sEMG), or simply EMG. Much research has gone into investigating the complicated relationship between EMG waveforms and joint torque [4]. Knowing that this relationship exists suggests that EMG is a useful measurement for assistive and rehabilitation robotic devices, and indeed myoelectric control interfaces have been designed for such devices since their early implementation [5]. We aim to design EMG-triggered rehabilitation exoskeletons for patients having an incomplete spinal cord injury (iSCI), a population that has historically seen less attention from researchers than, say stroke survivors due to their smaller numbers. Following recent efforts to characterize the EMG patterns of patients with iSCI for producing motion of either the hand [6], or the elbow and wrist [7], we wish to integrate this new knowledge into a myoelectric control system for the control of the MAHI Exo-II, an upper-limb rehabilitation exoskeleton for the elbow and wrist, to better serve the iSCI patient population.

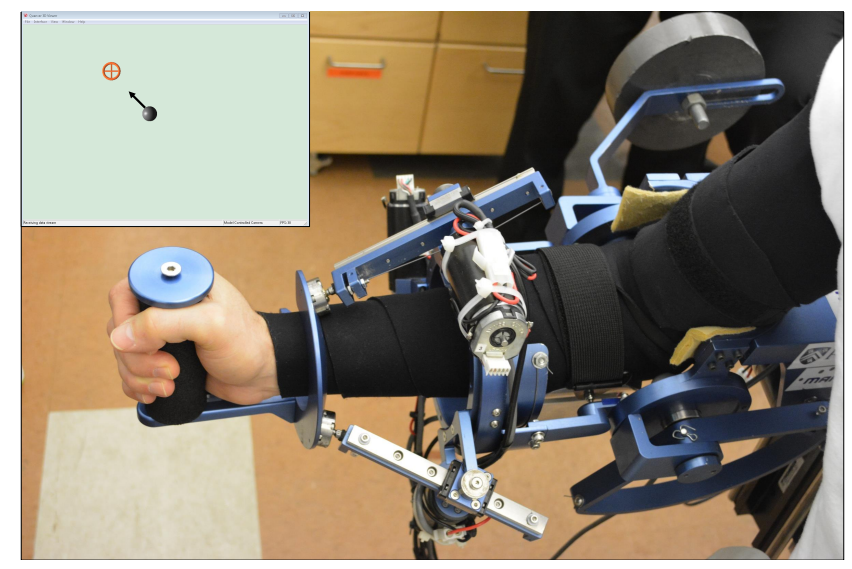

Fig. 1: MAHI Exo-II rehabilitation exoskeleton with the user's arm wrapped in a neoprene sleeve for insulation. The top-left insert is the target screen with the cursor that is displayed to the user.

The work presented in this paper is an exploration of the EMG characteristics of both healthy and iSCI subjects while wearing and operating the exoskeleton, Fig. 1. The subjects generated isolated single and multi degree-of-freedom (DoF) voluntary isometric contractions in sixteen possible directions. This work is a first step toward achieving EMG control of multi-DoF, coordinated movements of the upper-limb clinically viable implementations of such systems have yet to be produced in either exoskeletons or smart prostheses. We have demonstrated that by using a very small subset of the EMG time domain features, we can achieve average prediction sensitivity of $82 \%$ for healthy subjects and $66 \%$ for SCI subjects when selecting the intended direction of motion out of all sixteen single and multi-DoF possibilities. This performance was achieved using a commercial sEMG recording system with eight bipolar electrodes, suggesting that this number of electrodes and features may contain sufficient motor control information to support a rehabilitation 
protocol for iSCI patients utilizing myoelectric control of the robot in single and multi-DoF movements.

Powered assistive devices for the ankle [8], the hip and knee [9], the elbow [10], [11], and even shoulder, elbow, and wrist [12], [5] have all been designed and implemented with some form of myoelectric control. Most of these have been designed specifically for use in rehabilitation of persons following an injury, such as an iSCI. Though the goal of designers of rehabilitation robots, as well as the designers of smart prosthetics, is to achieve simultaneous and proportional control of multiple degrees of freedom of the arm and/or hand, creating such a system that is robust and reliable is still very much an open research question [13].

Problems such as inter-subject variability in the EMG signal are only amplified when considering a patient population such as SCI. In contrast, there has been considerable success in using pattern recognition (PR) techniques on EMG recordings of the muscles in the upper limb to recognize intended poses or directions of movement from patients with a wide variety of impairments [14], [15]. As it is our goal to give voluntary control of the exoskeleton to patients with a significant level of impairment, the identification of intended movements from EMG patterns is a worthwhile pursuit - even though it lacks the fluidity and possibility for continuous engagement that proportional control could provide, PR still gives users a sense of voluntary control over the limb while inside the exoskeleton. Though our stated goal is to utilize this EMG controller to assist individuals with no residual movement capability, the individuals with SCI who could be recruited to participate in this study could produce forces and motions detectable by the robot. Nevertheless, the SCI participant's muscle activity, as measured by sEMG, had been affected by the injury; therefore, by ignoring any residual motion we are simulating the desired situation. It should also be noted that the EMG based controller has significant advantage over the residual movement based controller because it can respond selectively to certain undesirable muscle activation patterns, e.g. significant cocontraction of the muscles, to which the movement based controller is blind.

The pattern recognition approach to myoelectric control is carried out in discrete steps. During a training phase subjects make voluntary contractions that are associated with predefined movements or poses, which constitute the user's intention. Next, certain features defined by the investigators are extracted from the recorded EMG waveforms for each observation of a user's voluntary contraction. A classification algorithm is then trained based on the extracted features and, in the case of supervised learning, the known labels of the user's intention. Parameters of the classifier are tuned according to the training data so that it may predict the user intention when presented with future observations of feature sets.

In [6], Liu and Zhou began investigating the motor control information that could be extracted from high-density surface EMG (HD-EMG) recordings on patients following incomplete cervical SCI. In their work they showed that indeed sufficient information could be extracted in order to predict different desired hand grasp patterns of the user, indicating that myoelectric pattern recognition techniques held potential for the control of assistive and rehabilitation devices for individuals with SCI. Their approach was to classify patterns using both the linear discriminant analysis (LDA) and knearest neighbor (KNN) classifiers, with results showing that both methods could achieve average overall classification accuracy higher than $97 \%$. Further investigation by Liu et al. into methods of classification were presented in [16], where it was shown that there is a great amount of redundancy amongst the many high-density electrode channels, as well as redundancy within the features of a single electrode channel. Therefore, a small fraction of the available features can be selected to classify the data with minimal losses in accuracy. This is useful for speed of training and implementation of classification algorithms, and additionally as a means to identifying the most relevant information contained in the EMG signal. In our work, we explore the problem of feature selection using the easily accessible built-in capabilities of MATLAB's Statistics and Machine Learning Toolbox, specifically, the neighborhood component analysis (NCA) method of feature selection [17].

Rojas-Martinez et al. have investigated the use of HDEMG spatial patterns in learning the intended movements of the elbow and wrist for both healthy subjects and subjects with cervical iSCI [18] [19]. They have shown the effectiveness of LDA and support vector machine (SVM) classifiers in predicting intended isometric motor tasks from individual subjects, and for the first time the possibility of groupspecific intention prediction for iSCI patients [7]. Though our current methods for intention classification are only subject specific, we present analysis of feature selection and feature distributions across subjects with the aim of generalizing to group-specific classification in the future.

Another significant difference between our methods and those discussed thus far is our use of a more conventional, commercial sEMG recording system with eight bipolar electrodes that we have placed over individual targeted muscles. One of the factors for this decision is that HDEMG recording arrays are still a relatively new technology and are more challenging to use effectively by those with limited expertise in the field of neurophysiology. In the same vein of reasoning, our ultimate goal is to develop a system that is as easily operated by a physical therapist as possible. It is therefore pertinent to ask what can be achieved by a traditional sEMG recording system that requires less intensive preparation and simpler data processing methods, despite the potential loss of information that is represented in the HD-EMG.

\section{METHODS}

We set out to quantify the sEMG patterns of activity of eight muscles in the upper limb during four single-DoF and two multi-DoF movements within our robotic exoskeleton. Five healthy subjects (4 male, 1 female, ages $22-50$ ) and two 
subjects with cervical-level iSCI (both male, injury level C5$\mathrm{C} 7$ incomplete, and at least 10 years post-injury) participated in this study. Using a selected subset of time-domain features of the EMG waveform, linear discriminant analysis (LDA) was used to classify the types of movements within singleDoF, multi-DoF, and combined datasets. LDA is a relatively simple algorithm that could be used to distinguish between the various DoFs and directions of motion for a future realtime controller, and it has become a standard approach in the literature.

\section{A. EMG-Equipped MAHI Exo-II}

The MAHI Exo-II is a robot exoskeleton designed for the rehabilitation of the elbow, forearm, and wrist [20]. It features capstan driven joints, serial elbow flexion and extension (EF/EE) and forearm pronation and supination (FP/FS) joints, and a parallel mechanism for wrist flexion and extension (WF/WE) and radial ulnar deviation (WR/WU). The robot is capable of outputting $7.35 \mathrm{Nm}$ of torque for the elbow DoF, $2.75 \mathrm{Nm}$ for the forearm, and $1.45 \mathrm{Nm}$ for both wrist axes. It features passive gravity compensation at the elbow joint. The MAHI Exo-II is equipped with optical encoders at each of the motors, from which we can extract position and velocity of all DoFs.

The Delsys Bagnoli EMG system provides us with eight channels of sEMG data. The variable gain for the channel amplification was set to 1000 . All data was read into our data acquisition system, the Quanser Q8-USB, sampled at $1 \mathrm{kHz}$.

\section{B. Experimental Protocol}

This experiment was designed to record the subject's sEMG signals for selected muscles during elbow, forearm, and wrist movements while they were held in an isometric pose by the exoskeleton. This analysis was performed for six different sets of DoFs. Four of these tests were done for single-DoF motions corresponding to elbow flexion and extension $(\mathrm{EF} / \mathrm{EE})$, forearm pronation and supination (FP/FS), wrist flexion and extension (WF/WE), and wrist radial and ulnar deviation (WR/WU). The other two tests were performed with multi-DoF combinations of these joints: combined elbow flexion and extension and forearm pronation and supination, and combining the two wrist DoFs.

Before the experiment, the height and shoulder abduction angle of the MAHI Exo-II were adjusted so the subject could hold their arm in a natural position with the elbow flexed while seated. The position of the chair relative to the exoskeleton was adjusted to keep both shoulders at equal heights and to keep the shoulder in the scapular plane $\left(\sim 30^{\circ}\right.$ from the frontal plane). Subjects were instructed not to move their torsos or shoulders during testing but restraints were not used to enforce this. The wrist handle location was positioned to provide a maximum range of motion while the subject held it in a natural grip. The elbow passive gravity compensation counterweight was set so that the subject was able to rest their elbow in the flexed position. The exoskeleton can be configured for left and right handed individuals, so the user's dominant arm was chosen for the experiment.
A Bagnoli-8 sEMG system (Delsys, Inc.) was used to collect sEMG signals from muscles that control the elbow, forearm, and wrist. The arm was first cleaned using isopropyl alcohol wipes. The upper half of the forearm and the lower half of the upper arm were shaved with a disposable razor to remove hair and dead skin. The target muscles for the sEMG electrodes were chosen as agonist-antagonist pairs for each of the DoFs. Muscles were chosen based on the experiment performed by Gopura et al. [21] as to minimize the noise and cross-talks associated with overlapping muscles. Muscles were located and palpated using guidelines enumerated in Table I. It should be noted that these muscles contribute to motion in multiple DoFs and not solely to the motion listed in the same row of Table I.

TABLE I: Selected Muscles and Corresponding Electrode Locations

\begin{tabular}{|c|c|c|}
\hline Movement & Muscle & $\begin{array}{l}\text { Location and } \\
\text { Palpation }\end{array}$ \\
\hline $\begin{array}{c}\text { Elbow } \\
\text { Flexion (EF) }\end{array}$ & $\begin{array}{l}\text { Biceps brachii } \\
\text { (BB) }\end{array}$ & $\begin{array}{l}\text { Front of the upper } \\
\text { arm, } 1 / 3 \text { of the way } \\
\text { up from the elbow }\end{array}$ \\
\hline $\begin{array}{c}\text { Elbow } \\
\text { Extension (EE) }\end{array}$ & $\begin{array}{l}\text { Triceps brachii } \\
\text { (TB) }\end{array}$ & $\begin{array}{c}\text { Posterior side of } \\
\text { the upper arm, } 1 / 3 \\
\text { of the way up from } \\
\text { the elbow }\end{array}$ \\
\hline $\begin{array}{c}\text { Forearm } \\
\text { Pronation (FP) }\end{array}$ & $\begin{array}{l}\text { Pronator teres } \\
(\mathrm{PT})\end{array}$ & $\begin{array}{l}\text { Medial side of the } \\
\text { cubital fossa, } \\
\text { pronating against } \\
\text { resistance }\end{array}$ \\
\hline $\begin{array}{c}\text { Forearm } \\
\text { Supination (FS) }\end{array}$ & $\begin{array}{l}\text { Supinator } \\
\text { (S) }\end{array}$ & $\begin{array}{l}\text { Outside of the } \\
\text { forearm below the } \\
\text { lateral epicondyle } \\
\text { supinating against } \\
\text { resistance }\end{array}$ \\
\hline $\begin{array}{c}\text { Wrist } \\
\text { Flexion (WF) }\end{array}$ & $\begin{array}{l}\text { Flexor carpi } \\
\text { radialis } \\
\text { (FCR) }\end{array}$ & $\begin{array}{l}\text { On the forearm } \\
\text { below the medial } \\
\text { epicondyle by } \\
\text { flexing and radial } \\
\text { deviation of the wrist }\end{array}$ \\
\hline $\begin{array}{c}\text { Wrist } \\
\text { Extension (WE) }\end{array}$ & $\begin{array}{l}\text { Extensor carpi } \\
\text { ulnaris } \\
\text { (ECU) }\end{array}$ & $\begin{array}{c}\text { Lateral side of the } \\
\text { forearm, extending } \\
\text { and ulnar deviation of } \\
\text { the wrist }\end{array}$ \\
\hline $\begin{array}{c}\text { Wrist Radial } \\
\text { Deviation (WR) }\end{array}$ & $\begin{array}{l}\text { Extensor carpi } \\
\text { radialis longus } \\
\text { (ECRL) }\end{array}$ & $\begin{array}{l}\text { Top of the forearm } \\
\text { lateral of the } \\
\text { brachialis, extending } \\
\text { and radial deviation } \\
\text { of the wrist }\end{array}$ \\
\hline $\begin{array}{c}\text { Wrist Ulnar } \\
\text { Deviation (WU) }\end{array}$ & $\begin{array}{l}\text { Flexor carpi } \\
\text { ulnaris } \\
\text { (FCU) }\end{array}$ & $\begin{array}{l}\text { Bottom of the } \\
\text { forearm medial to } \\
\text { the ulna, felt during } \\
\text { ulnar deviation of } \\
\text { the wrist }\end{array}$ \\
\hline
\end{tabular}

Once inside the exoskeleton the subject was strapped to the robot at the upper forearm and the hand. The neutral position of the robot handle was adjusted to match the length of the subject's forearm, and the neutral orientation of the handle was always set to the mid-pronated position and perpendicular to the forearm. The subject was instructed to place the back of their upper-arm in contact with the pad on the back of the exoskeleton. Neoprene wrapping was 

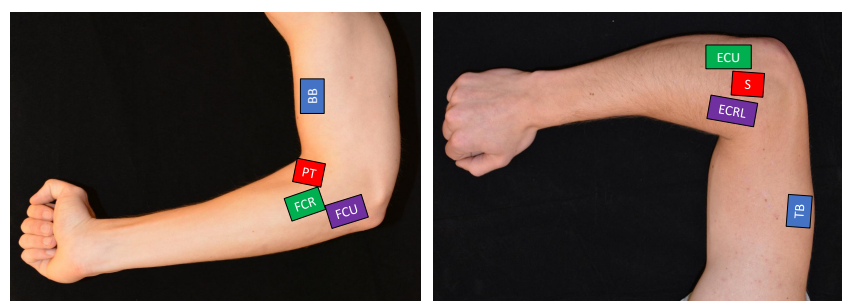

Fig. 2: Approximate locations for each sEMG electrode labeled by muscle. Following the organization of Table I, muscles associated with the movement EF/EE are labeled in blue, FP/FS in red, WF/WE in green, and WR/WU are labeled in purple.

used to insulate the sEMG electrodes from the metal of the exoskeleton and the electrical interference from the motors.

Subjects completed blocks of trials with 1 min breaks in between each block of movements. Each block involved only one set of the single or multi-DoF movements. Before testing, subjects were presented with practice sessions for each DoF to familiarize themselves with the exoskeleton motion and timing of the isometric contractions. For this experiment the subjects were instructed to direct effort along a specified DoF while the robot held their arm in an isometric pose. There were three different isometric poses for each DoF: one at the upper end of the range of motion, one a the midpoint, and one at the lower end of the range of motion. A flashing target and an arrow pointing from it prompted the subject to initiate effort in that direction. Following a 2-second contraction window, the user was moved by the exoskeleton to the next isometric position over an approximately 2-second transition, given 1 second of rest in the new position, and then started the next 2second contraction window. Users were instructed to remain passive as the exoskeleton moved between isometric positions meaning they should limit voluntary contractions but no steps were taken to enforce this condition. Each subject was presented with fifteen repetitions of each direction of a reaching movement in a pseudo-random order. With two directions per single-DoF motion and four directions per multi-DoF movement, this resulted in 60 and 120 trials per block respectively.

\section{Data Analysis}

The eight EMG channels all went through the same postprocessing steps that have now become almost standard for sEMG data, namely, band-pass filtering at 20-450 Hz (fourthorder butterworth filter) and removal of the mean. The data from each electrode was also normalized by dividing by the maximum voltage recorded on that electrode throughout the entire session, producing a signal referred to as the electrode excitation. This was chosen instead of normalizing to the maximum voluntary contraction because the maximum voluntary contraction value is highly dependent on the task designed to measure it, and can potentially be exceeded during experiments.
Data collected from all channels was segmented according to the automated cues provided to the user through the visual interface. Each isometric contraction took place within a 2-second window, within which a $200 \mathrm{~ms}$ window was identified as the time of constant voluntary contraction. This smaller window was identified from the user's joint torque in the appropriate DoF, as estimated by the robot's commanded motor currents. This torque measurement also served as a check to ensure that user's were producing contractions in accordance with the on-screen targets and guard against mislabelling. The segmented data was used to isolate time-domain features from the sEMG waveforms. The time domain features that were collected were the normalized root-mean square (nRMS), normalized mean absolute value (nMAV), normalized waveform length (nWL), normalized number of zero crossings (nZC), normalized number of slope sign changes (nSSC), and the four-order autocorrelation coefficients (AR1, AR2, AR3, AR4). Data was partitioned for 10 -fold cross validation and the sEMG time-domain features were analyzed and selected to minimize the classification error using the MATLAB function fscnca().

Using the selected features, classification of movements based on EMG features was performed using the built-in MATLAB linear discriminant analysis (LDA) algorithms. The prediction error was evaluated by using holdout evaluation to randomly divide the features and labels into a training and testing set. The training set was $80 \%$ of the total data with the remaining $20 \%$ set aside for evaluating prediction accuracy. The selected features with cross-validation error and the prediction error from the LDA were reported to asses the feasibility of developing a real-time controller for a population of subjects based on that scheme.

\section{RESULTS \& DISCUSSION}

\section{A. sEMG Feature Selection}

There were eight channels of sEMG data corresponding to eight different muscles and 9 time domain features (nRMS, nMAV, nWL, nZC, nSSC, AR1, AR2, AR3, AR4) totaling 72 features for each movement. To identify the features that show distinct differences depending on the direction of movement (class) a Neighborhood Component Analysis (NCA) was used. This analysis was performed in isolation for each subject, creating a subject-specific set of features to be used for classification. For each subject, the NCA analysis was performed for three different groups: all singleDoF motions, all multi-DoF motions, and the combined data from all the single and multi-DoF motions. For singleDoF classification, the algorithm identified an average of 10 relevant features for each subject to be used for classification. For multi-DoF classification, the algorithm identified an average of 11 relevant features for each subject to be used for classification. For the combined data the algorithm identified an average 17 features for each subject to be used for classification. The subject-specific sets of features were used for the classification algorithm shown in Section III-C. The selected features for single-DoF and multi-DoF datasets are shown across all subjects in Fig. 3. 


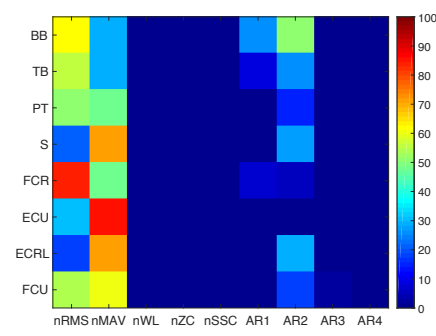

(a) Single DoF

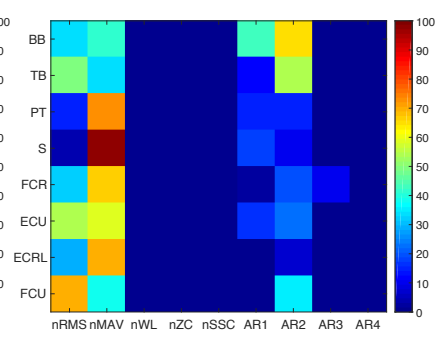

(b) Multi DoF
Fig. 3: Frequency of selection for features using the NCA algorithm for single and mutli-DoF movement classification. Color indicates the percentage of subjects for which a certain feature was chosen out of all subjects. Feature descriptions are given in subsection II-C.

The selected features were narrowed further based on the frequency at which they were selected by the NCA algorithm. A threshold of being selected at least $70 \%$ of the time was applied to select a set of features for comparison across populations. This threshold resulted in four features identified to be used to classify within the single-DoF group, four for the multi-DoF group, and seven features for the combined group. These identified features were used to compare the healthy and SCI populations in Section III-B. The main features that were selected are nRMS, nMAV, and AR. If a real-time controller is implemented, nWL, nZC, and $\mathrm{nSSC}$ could be disregarded to improve speed because they were never selected by the classifier. It should be noted that nRMS and nMAV are somewhat similar in meaning, both being a measure of the EMG amplitude. Additionally, most of the features selected pertained to motion in the forearm and wrist. The wrist and forearm are active in elbow movements as well to stabilize the wrist on the handle. This could be why muscles that primarily flex and extend the elbow do not seem to be as significant for classification.

\section{B. sEMG Feature Comparison}

Multi-DoF data for elbow/forearm and wrist modes are presented in Fig. 4. The features shown in the plot were the narrowed NCA features selected from the combined dataset. An average of each feature across all observations of a specific movement was taken for each subject. The data shown in Fig. 4 is a distribution of the means for each features for the healthy population. The means for each feature for both SCI subjects are shown as purple and green dots.

It can be seen in Fig. 4 that the nMAV is higher for muscles mapped to a motion while the subjects are performing that motion (i.e. high S MAV during supination movements). This helps to validate our methods in mapping muscles to motions and our electrode placement scheme. High variability within the means of features was observed among healthy population. This is in part because we did not include a way to monitor or control user effort. A force and torque sensor will be integrated in the exoskeleton handle for future studies to monitor this. Some variability is still anticipated, but equalizing the users' effort levels should make these values less variable.

In some instances the means for the two SCI subject's features are well outside of of the healthy population's distribution. This seems to happen more for the pronator teres and supinator muscle groups. This could be due to the difficulty of palpating these smaller muscles for this population, a higher loss of function in these specific areas, or a combination of these factors. Co-contraction could be possible as well (i.e. high PT nMAV during supination).

The two SCI subjects compared closer to the healthy distribution for wrist movements and were more commonly within one standard deviation of the healthy population's mean. This could potentially be due to the lower effort levels required to reach these targets.

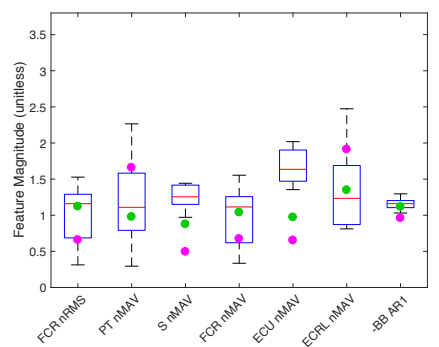

(a) WF-WR

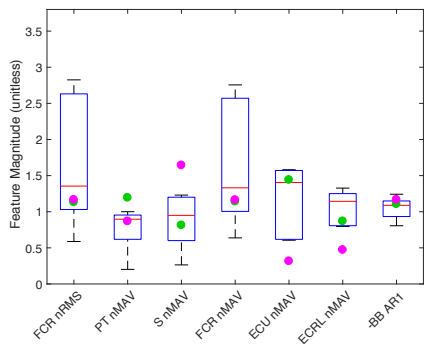

(c) WF-WU

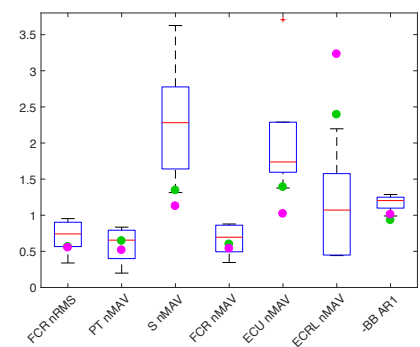

(b) WE-WR

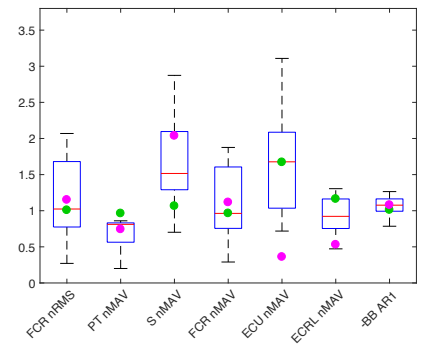

(d) WE-WU
Fig. 4: Magnitude of selected features - averaged across trials - for four different wrist multi-DoF movements that are combinations of movements listed in Table I. Box plots represent distributions of healthy subject data, and SCI subjects 1 and 2 are shown as purple and green dots, respectively.

\section{Classification}

The performance of the classifier using the subject-specific NCA selected features is shown in Fig. 5, and was evaluated using the approach in [7].

Overall, the classifier performed better for the healthy population than the SCI population, as might be expected. Accuracy was very high for all groups, making an LDA classifier a strong candidate for use in future exoskeleton controllers. The classification sensitivity was $82 \%$ for the healthy population for selecting the intended direction of motion from 16 possible motions (the most challenging classification task), and sensitivity was $66 \%$ for the two SCI subjects for a set of the same 16 possible motions. 


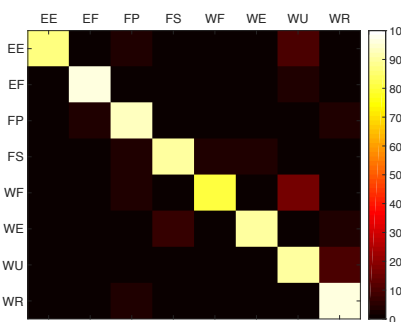

(a) Single DoF Healthy

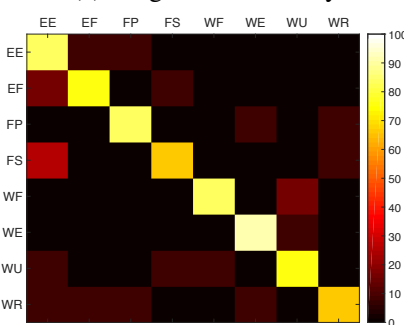

(c) Single DoF SCI

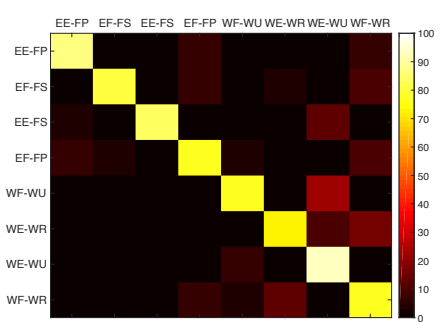

(b) Multi DoF Healthy

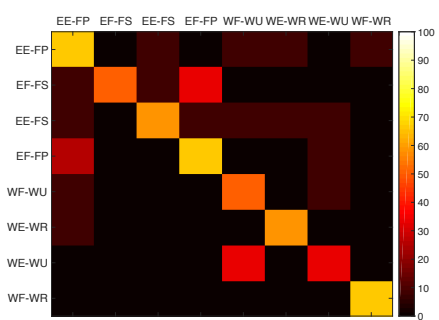

(d) Multi DoF SCI
Fig. 5: Classification performance across subjects is reported as a confusion matrix for single and multi-DoF motion classifiers in both healthy participants and those with SCI. Color indicates the percentage of positive classifications out of 6 total instances of each class in the testing data set.

\section{CONCLUSIONS}

Our integrated robotic exoskeleton and sEMG recording system for rehabilitation following SCI has been shown to accurately predict the intended single and multi-DoF movements of both healthy and SCI subjects using a very small subset of the EMG waveform features extracted from only eight bipolar electrodes. This suggests that eight sEMG channels may contain sufficient motor control information to support a rehabilitation protocol for iSCI patients utilizing myoelectric control of the robot in single and multi-DoF movements, without having to resort to high-density EMG arrays. A subset of amplitude features and autocorrelation features from the EMG signal have been identified as particularly useful for this classification task. Our future work will be to evaluate the performance of an LDA classifier that is trained on an entire group, potentially alleviating the need for intensive classifier training. In order to improve the consistency of our EMG data collection, we will be incorporating force/torque sensing into the robotic exoskeleton. In the future, we will work with more iSCI subjects to evaluate the efficacy of a real-time EMG-controlled exoskeleton for rehabilitation.

\section{REFERENCES}

[1] R. Riener, T. Nef, and G. Colombo, "Robot-aided neurorehabilitation of the upper extremities," Medical and biological engineering and computing, vol. 43, no. 1, pp. 2-10, 2005.

[2] D. J. Reinkensmeyer, J. L. Emken, and S. C. Cramer, "Robotics, motor learning, and neurologic recovery," Annu. Rev. Biomed. Eng., vol. 6, pp. 497-525, 2004.
[3] N. Yozbatiran, J. Berliner, M. K. O’Malley, A. U. Pehlivan, Z. Kadivar, C. Boake, and G. E. Francisco, "Robotic training and clinical assessment of upper extremity movements after spinal cord injury: a single case report," Journal of rehabilitation medicine, vol. 44, no. 2, pp. 186-188, 2012.

[4] E. A. Clancy and N. Hogan, "Relating agonist-antagonist electromyograms to joint torque during isometric, quasi-isotonic, nonfatiguing contractions," IEEE Transactions on Biomedical Engineering, vol. 44, no. 10, pp. 1024-1028, 1997.

[5] L. Dipietro, M. Ferraro, J. J. Palazzolo, H. I. Krebs, B. T. Volpe, and N. Hogan, "Customized interactive robotic treatment for stroke: Emg-triggered therapy," IEEE Transactions on Neural Systems and Rehabilitation Engineering, vol. 13, no. 3, pp. 325-334, 2005.

[6] J. Liu and P. Zhou, "A novel myoelectric pattern recognition strategy for hand function restoration after incomplete cervical spinal cord injury," IEEE Transactions on Neural Systems and Rehabilitation Engineering, vol. 21, no. 1, pp. 96-103, 2013.

[7] M. Jordanić, M. Rojas-Martínez, M. A. Mañanas, and J. F. Alonso, "Prediction of isometric motor tasks and effort levels based on highdensity emg in patients with incomplete spinal cord injury," Journal of neural engineering, vol. 13, no. 4, p. 046002, 2016.

[8] S. Au, M. Berniker, and H. Herr, "Powered ankle-foot prosthesis to assist level-ground and stair-descent gaits," Neural Networks, vol. 21, no. 4, pp. 654-666, 2008.

[9] B. Shen, J. Li, F. Bai, and C.-M. Chew, "Motion intent recognition for control of a lower extremity assistive device (lead)," in Int. Conf. on Mechatronics and Automation. IEEE, 2013, pp. 926-931.

[10] T. Lenzi, S. M. M. De Rossi, N. Vitiello, and M. C. Carrozza, "Intention-based emg control for powered exoskeletons," IEEE Trans. on Biomedical Engineering, vol. 59, no. 8, pp. 2180-2190, 2012

[11] K. Kiguchi, S. Kariya, K. Watanabe, K. Izumi, and T. Fukuda, "An exoskeletal robot for human elbow motion support-sensor fusion, adaptation, and control," IEEE Transactions on Systems, Man, and Cybernetics, Part B (Cybernetics), vol. 31, no. 3, pp. 353-361, 2001.

[12] K. Kiguchi and Y. Hayashi, "An emg-based control for an upper-limb power-assist exoskeleton robot," IEEE Trans. on Systems, Man, and Cybernetics, Part B (Cybernetics), vol. 42, no. 4, pp. 1064-1071, 2012.

[13] F. Lunardini, C. Casellato, A. d'Avella, T. D. Sanger, and A. Pedrocchi, "Robustness and reliability of synergy-based myocontrol of a multiple degree of freedom robotic arm," IEEE Transactions on Neural Systems and Rehabilitation Engineering, vol. 24, no. 9, pp. 940-950, 2016.

[14] P. Zhou, M. M. Lowery, K. B. Englehart, H. Huang, G. Li, L. Hargrove, J. P. Dewald, and T. A. Kuiken, "Decoding a new neuralmachine interface for control of artificial limbs," Journal of neurophysiology, vol. 98, no. 5, pp. 2974-2982, 2007.

[15] S. W. Lee, K. M. Wilson, B. A. Lock, and D. G. Kamper, "Subjectspecific myoelectric pattern classification of functional hand movements for stroke survivors," IEEE Transactions on Neural Systems and Rehabilitation Engineering, vol. 19, no. 5, pp. 558-566, 2011.

[16] J. Liu, X. Li, G. Li, and P. Zhou, "Emg feature assessment for myoelectric pattern recognition and channel selection: A study with incomplete spinal cord injury," Medical engineering \& physics, vol. 36, no. 7, pp. 975-980, 2014.

[17] W. Yang, K. Wang, and W. Zuo, "Neighborhood component feature selection for high-dimensional data." JCP, vol. 7, no. 1, pp. 161-168, 2012.

[18] M. Rojas-Martínez, M. A. Mañanas, and J. F. Alonso, "High-density surface emg maps from upper-arm and forearm muscles," Journal of neuroengineering and rehabilitation, vol. 9, no. 1, p. 85, 2012.

[19] M. Jordanic, M. Rojas-Martínez, M. A. Mañanas, and J. F. Alonso, "Spatial distribution of hd-emg improves identification of task and force in patients with incomplete spinal cord injury," Journal of neuroengineering and rehabilitation, vol. 13, no. 1, p. 41, 2016.

[20] K. D. Fitle, "Clinical evaluation of an upper limb exoskeleton for rehabilitation after incomplete spinal cord injury," Ph.D. dissertation, Rice University, 2015.

[21] R. A. R. C. Gopura and K. Kiguchi, "Emg-based control of an exoskeleton robot for human forearm and wrist motion assist," in 2008 IEEE International Conference on Robotics and Automation, May 2008, pp. 731-736. 